\title{
Sirtuin 1 participates in intervertebral disc degeneration via the nicotinamide phosphoribosyl transferase/nicotinamide adenine dinucleotide/sirtuin 1 pathway responsible for regulating autophagy of nucleus pulposus cells
}

\author{
QIFENG SHI, LIANG XU and XIANGYI ZENG
}

Department of Orthopedics, The Third Affiliated Hospital of Jinzhou Medical University, Jinzhou, Liaoning 121000, P.R. China

Received August 1, 2020; Accepted March 10, 2021

DOI: $10.3892 /$ etm.2022.11193

\begin{abstract}
Disc degeneration is the main cause of discogenic low back pain, disc herniation, degenerative stenosis of spinal canal, lumbar spondylolisthesis and other diseases. In the process of intervertebral disc degeneration, water and extracellular matrix of nucleus pulposus tissues are lost, so the normal tension in the intervertebral disc cannot be maintained, which worsens the living environment of nucleus pulposus cells. Low back pain (LBP), with a high incidence rate of disability, has become an increasing health concern and a social and economic problem. The present study aimed to analyze the action mechanisms of nicotinamide phosphoribosyl transferase (Nampt) and sirtuin 1 (SIRT1) in intervertebral disc degeneration (IVDD). In total 26 patients with lumbar disc herniation who had surgical resection at The Third Affiliated Hospital of Jinzhou Medical University were recruited as the experimental group and their degenerative nucleus pulposus (DNP) tissues of intervertebral disc were collected. In addition, nucleus pulposus tissues of intervertebral disc were collected from 20 patients with burst fracture of lumbar spine at the same hospital (control). Nucleus pulposus cells from primary culturing were separated for subsequent experimentation. LC3 II/I, beclin-1, SIRT1 and NAMPT mRNA and protein expression levels were determined using reverse transcription-quantitative PCR and western blotting, respectively. Nicotinamide adenine dinucleotide (NAD) contents in nucleus pulposus cells was determined by NAD assay kit. The mRNA and protein expression levels of SIRT1 in DNP tissues were reduced compared with the control tissues and decreased with increasing disease severity. The expression of autophagy-associated LC3 II/I and beclin-1 in DNP tissues
\end{abstract}

Correspondence to: Dr Xiangyi Zeng, Department of Orthopedics, The Third Affiliated Hospital of Jinzhou Medical University, 2 Heping Road, Jinzhou, Liaoning 121000, P.R. China

E-mail: fszxy2009@163.com

Key words: intervertebral disc degeneration, nucleus pulposus, nicotinamide phosphoribosyl transferase, sirtuin 1, LC3, beclin-1s was reduced compared with control tissues. SIRT1 regulated the LC3 II/I and beclin-1 expression levels in nucleus pulposus cells. Treatment with resveratrol and inhibitor of SIRT1 showed that Nampt/NAD $/$ SIRT1 pathway participated in the process of IVDD by regulating autophagy of nucleus pulposus cells. SIRT1 serves a role in the process of IVDD through Nampt/NAD ${ }^{+} /$SIRT1 pathway that regulates autophagy of nucleus pulposus cells. SIRT1 may become a biological target for the treatment of IVDD.

\section{Introduction}

Back and waist pain, especially low back pain (LBP), has become an increasing human health problem due to its high incidence (20\%) and high disability rate (1). The cause and mechanism of LBP are not clear. At present, it is believed that LBP is closely related to intervertebral disc degeneration (IVDD) (2,3). A complete intervertebral disc is composed of the annulus fibrosus, nucleus pulposus and cartilage endplate and the reason of IVDD may be the pathological process of tissue degeneration and progressive structural destruction of nucleus pulposus cells induced by overload (4). The main causes of IVDD include mechanical stress of the spine, inflammation, aging, biochemical factors such as radiation and genetic and racial factors $(5,6)$. At present, the main treatment methods for IVDD include conservative anti-inflammatory and analgesic therapies, physical therapy and surgical discectomy and replacement of intervertebral discs (3). However, these methods are not effective enough for IVDD, especially IVDD at early and middle stages that has no surgical indication $(2,7)$. Hence, understanding the pathogenesis of IVDD and methods to delay or even inhibit IVDD are becoming hot topics for research.

Sirtuin 1 (SIRT1) belongs to the mammalian Sirtuin protein family (SIRT1, SIRT2, SIRT3, SIRT4, SIRT5, SIRT6 and SIRT7) that are type III histone deacetylases (HDACs) (8). Type III HDACs are distinct from type I and type II HDACs as the activation of type III HDACs is dependent on nicotinamide adenine dinucleotide $\left(\mathrm{NAD}^{+}\right)$(9). The substrates of SIRT1 include not only histone, but also other transcription factors and synergistic factors, such as p53, E2F transcription factor 1 (E2F1), peroxisome proliferators-activated receptor- $\gamma(\operatorname{PPAR}-\gamma)$ 
and Forkhead box O3 (FOXO) (8). Hence, SIRT1 is an important factor involved in growth, development, cell metabolism and aging $(10,11)$. The expression of SIRT1 in articular chondrocytes of patients with osteoarthritis is significantly lower compared with that in healthy people, while the expression of apoptosis-related caspase- 3 and caspase- 9 proteins is increased and the apoptotic rate is enhanced after interfering with the expression of SIRT1 using silencing transfection experiments (12). SIRT1 plasmid lentiviral transfection into osteoarthritis chondrocytes leads to the overexpression of SIRT1 which significantly increases the amount of extracellular matrix $(13,14)$. At the early stage of multiple myeloma, SIRT1 may promote autophagy of multiple myeloma cells and participate in the promotional effect of autophagy on cell survival $(15,16)$.

Nicotinamide phosphoribosyl transferase (Nampt) is a rate-limiting enzyme for the $\mathrm{NAD}^{+}$remedial synthetic pathway in mammalian cells (17). Nampt is widely expressed in various tissues and cells such as brain and liver, and participates in the regulation of important physiological and pathological processes in cells, such as cell differentiation, stress response, cell apoptosis, etc. $(18,19)$. The content of Nampt in serum and joint fluid of patients with osteoarthritis is significantly higher compared with that of normal people (20). Intraarticular injection of adenovirus overexpressing Nampt can lead to cartilage destruction and osteoarthritis, whereas intraarticular administration of AP0866, a Nampt inhibitor can reduce the inflammatory response in osteoarthritis (21). A positive association exists between the serum level of Nampt in patients with rheumatoid arthritis and the degree of inflammation and disease (22). Nampt can activate the transcription factor $\mathrm{NF}-\kappa \mathrm{B}$ in fibroblasts in the synovial fluid of patients with rheumatoid arthritis, which induces the expression of cytokines interleukin (IL)-6, IL-8 and matrix metalloproteinase (MMP)-1 (23). To the best of our knowledge, to date there is no report on the specific regulatory mechanism of Nampt in IVDD.

The present study analyzed the mechanisms of Nampt and SIRT1 in IVDD and tested whether SIRT1 could become a biological target for the treatment of IVDD.

\section{Materials and methods}

Subjects. In the present study, the experimental group consisted of 26 patients with IVDD (12 males and 14 females; mean age, $55 \pm 3.2$ years) who had surgical resection and their DNP tissues of intervertebral disc were collected at The Third Affiliated Hospital of Jinzhou Medical University in March 2019 (Jinzhou, China). The control group from the same hospital consisted of 20 patients with burst fracture of lumbar spine (11 males and 9 females; mean age, $49 \pm 4.3$ years) and their nucleus pulposus tissues of intervertebral disc were collected by posterior decompression, discectomy, bone graft fusion and internal fixation within $24 \mathrm{~h}$ after injury. All patients with a history of low back pain were excluded. When collecting the control samples, patients with intervertebral disc lesions were excluded to ensure the reliability of the control samples. All patients were examined by MRI before operation. IVDD was classified according to Pfirrmann classification (7). Grade II and grade III degenerations were considered as mild degeneration and grade IV and grade $\mathrm{V}$ degenerations were considered as severe degeneration (7). The present study was approved by the Ethics Committee of Jinzhou Medical University (Jinzhou, China). In addition, written informed consent was provided by all patients and/or their family members. Degenerative disc nucleus pulposus tissues originated from patients who had spinal degenerative diseases earlier and received conventional posterior decompression, discectomy, bone graft fusion and internal fixation.

Cells and treatment. Nucleus pulposus tissues of intervertebral disc from both groups were separated and cut into $1 \mathrm{~mm}^{3}$ pieces. The tissues were washed with PBS for 3 times to remove blood and then $2 \mathrm{U} / \mathrm{ml}$ trypsin (Thermo Fisher Scientific, Inc.) was added to the samples prior to centrifugation at $1,500 \mathrm{x} \mathrm{g}$ and $4^{\circ} \mathrm{C}$ for $5 \mathrm{~min}$. Tissues were digested with $0.25 \mathrm{mg} / \mathrm{ml}$ type II collagenase (Sigma-Aldrich; Merck KGaA) for $4 \mathrm{~h}$ at $37^{\circ} \mathrm{C}$ until the tissue masses disappeared. After filtration through a $40-\mu \mathrm{m}$ pore, the samples were centrifuged again at $800 \mathrm{x} \mathrm{g}$ for $5 \mathrm{~min}$ at room temperature and the supernatant was discarded. Subsequently, nucleus pulposus cells were re-suspended with DMEM/F12 medium (Thermo Fisher Scientific, Inc.) containing 10\% FBS (Thermo Fisher Scientific, Inc.) supplemented with $100 \mathrm{U} / \mathrm{ml}$ penicillin and streptomycin. After counting, cell culture was conducted in a $37^{\circ} \mathrm{C}, 5 \% \mathrm{CO}_{2}$ incubator for 3 days.

Nucleus pulposus cells were seeded into 6-well plates (Corning, Inc.) at a density of $8 \times 10^{4}$. The whole experiment was carried out at room temperature. The nucleus pulposus of adult intervertebral disc is mainly chondroid nucleus pulposus cells, and its extracellular matrix is mainly glycosaminoglycans, proteoglycan and type II collagen. In the process of intervertebral disc degeneration, the water and extracellular matrix of nucleus pulposus are lost, which destroys the microenvironment of nucleus pulposus cells. To investigate the mechanism by which SIRT1 (Sigma-Aldrich; Merck KGaA) regulates IVDD, nucleus pulposus cells were treated with SIRT1 agonist resveratrol $(8 \mu \mathrm{mol} / \mathrm{l}$; Sigma-Aldrich; Merck KGaA) or SIRT1 inhibitor nicotinamide (12 mmol/l; Sigma-Aldrich; Merck KGaA) and SIRT1, LC3 II/I and beclin-1 expression levels were determined because these factors are associated with autophagy $(8,15)$. To explore the mechanism of action of Nampt and SIRT1 in IVDD, Nampt inhibitor FK866 (25 $\mu \mathrm{mol} / \mathrm{l}$; Sigma-Aldrich; Merck $\mathrm{KGaA}$ ) was used to treat nucleus pulposus cells.

Reverse transcription-quantitative $(R T-q) P C R$. Nucleus pulposus tissue was lysed with TRIzol ${ }^{\circledR}$ (Invitrogen; Thermo Fisher Scientific Inc.) prior to obtaining total RNA by the phenol chloroform method (8). After quantification with the Nanodrop ND2000 ultraviolet spectrophotometry (Thermo Fisher Scientific, Inc.), RNA (1 $\mu \mathrm{g})$ was subjected to reverse transcription using the TIANScript II cDNA First Strand Synthesis kit (Tiangen Biotech Co., Ltd.) to obtain cDNA according to the manufacturer's manual, which was then stored at $-20^{\circ} \mathrm{C}$. RT-qPCR was performed using the SuperReal PreMix RT-qPCR kit (SYBR Green; Tiangen Biotech Co., Ltd.) on the iQ5 PCR instrument (Bio-Rad Laboratories Inc.). The primers used were as follows: SIRT1 forward, 5'-CAGCCGTCTCTGTGTC ACAAA-3' and reverse, 5'-GCACCGAGGAACTACCTGAT-3'; LC3 forward, 5'-CTCCCAGAAACAACGG-3' and reverse, 
5'-GCCTACATTCAGTGCC-3'; beclin-1 forward, 5'-TCTA CACGCTACACCC-3' and reverse, 5'-AAGACCTCACCC TCC-3'; Nampt forward, 5'-GCCAGCACCACCAATGTA AGT-3' and reverse, 5'-GGGGTCAATCCAGTAGTCTC CAC-3'; and GAPDH forward, 5'-TCCCATCACCATCTT-3' and reverse, 5'-TCCCATCACCATCTT-3'. The RT-qPCR system $(20 \mu \mathrm{l})$ contained $2 \mu \mathrm{l}$ cDNA, $0.5 \mu \mathrm{l}$ upstream primer, $0.5 \mu \mathrm{l}$ downstream primer, $10 \mu \mathrm{l}$ SYBR Premix Ex Taq and $7 \mu \mathrm{ldd} \mathrm{H}_{2} \mathrm{O}$. The thermocycling used were as follows: $95^{\circ} \mathrm{C}$ for $5 \mathrm{~min}$; a total of 46 cycles of $95^{\circ} \mathrm{C}$ for $20 \mathrm{sec}$ and $55^{\circ} \mathrm{C}$ for $20 \mathrm{sec}$; followed by $72^{\circ} \mathrm{C}$ for $30 \mathrm{sec}$. Each experiment was performed in triplicate. Relative mRNA expression levels of SIRT1 were determined using the $2^{-\Delta \Delta C q}$ method (9) and were normalized to the internal control GAPDH.

Western blotting. Western blotting was performed according to a previous report (7). Briefly, totally $100 \mathrm{mg}$ experimental and control tissues were lysed with $600 \mu \mathrm{l}$ RIPA lysis buffer (Beyotime Institute of Biotechnology) on ice for $30 \mathrm{~min}$. After centrifugation at $12,000 \mathrm{x} \mathrm{g}$ at $4^{\circ} \mathrm{C}$ for $10 \mathrm{~min}$, the supernatant was collected. After concentration determination with the RTP7102 bicinchoninic acid (BCA) kit [Real-Times (Beijing) Biotechnology Co., Ltd.], 5X SDS loading buffer was added into the samples, which were then boiled for $5 \mathrm{~min}$. Protein sample $(20 \mu \mathrm{g} / \mathrm{lane})$ was subjected to $10 \%$ SDS-PAGE. Protein samples were then electronically transferred onto polyvinylidene difluoride membranes on ice. After being blocked with $5 \%$ skimmed milk at room temperature, the blots were incubated with rabbit anti-human anti-SIRT1 (1:1,000; cat. no. ab189494), anti-LC3 (1:1,000; cat. no. ab192890), anti-beclin-1 (1:1,000; cat. no. ab254511) or GAPDH (1:1,000; cat. no. ab9485) polyclonal primary antibodies (all from Abcam) at $4^{\circ} \mathrm{C}$ overnight. The membrane was washed with $0.1 \%$ Tween-20-containing PBS for 3 times, the blots were then treated with corresponding goat anti-rabbit horseradish peroxidase-conjugated secondary antibodies (1:1,000; cat. no. ab205718; Abcam) at $4^{\circ} \mathrm{C}$ for $1 \mathrm{~h}$. Enhanced chemiluminescence (ECL) method (Abcam) was performed for color development. Protein bands were analyzed using Image Lab v.3.0 software (Bio-Rad Laboratories Inc.). Relative expression levels of target proteins were normalized to GAPDH.

Determination of NAD contents within nucleus pulposus cells of the control group. For the extraction of $\mathrm{NAD}^{+}$, nucleus pulposus cells $\left(10^{4}\right)$ were mixed with $1 \mathrm{ml}$ acidic extraction solution (Sciencell Research Laboratories Inc.) and lysed with ultrasound. After being incubated in a water bath at $95^{\circ} \mathrm{C}$ for $5 \mathrm{~min}$, the samples were subjected to centrifugation at $10,000 \mathrm{x}$ g at $4^{\circ} \mathrm{C}$ for $10 \mathrm{~min}$. In total $0.5 \mathrm{ml}$ supernatant was mixed with $0.5 \mathrm{ml}$ alkaline extraction liquid (cat. no. ab65348; Abcam). After being centrifuged again at $12,000 \mathrm{xg}$ and room temperature for $10 \mathrm{~min}$, the supernatant sample was collected and placed on ice until use.

The absorbance of the samples was read at $570 \mathrm{~nm}$ and NAD contents were calculated based on the manufacturer's instructions of the NAD assay kit (Sciencell Research Laboratories Inc.).

Statistical analysis. Data in this study were presented as means \pm standard deviations. Statistical analysis was conducted using SPSS 22.0 software (IBM Corp.). Comparison between
2 groups was performed using unpaired Student's t-test. Multiple group comparison was conducted using one-way analysis of variance, followed by the post hoc Student-Newman-Keuls test. All tests were performed for 3 times. $\mathrm{P}<0.05$ was considered to indicate a statistically significant difference.

\section{Results}

SIRT1 expression levels in DNP tissues are reduced compared with control tissues and decrease with increasing disease severity. To examine SIRT1 expression levels in DNP and control tissues, mRNA and protein expression levels were detected using RT-qPCR and western blotting, respectively. SIRT1 mRNA and protein expression level in DNP tissues were significantly elevated compared with control tissues $(\mathrm{P}<0.05$; Fig. $1 \mathrm{~A}$ and $\mathrm{B})$. In addition, mRNA and protein expression levels of SIRT1 in DNP tissues from patients at grades IV and $\mathrm{V}$ were significantly decreased compared with DNP tissues from patients at grades I, II and III $(\mathrm{P}<0.01$; Fig. 1C and D). Taken together, the aforementioned results suggested that SIRT1 expression levels are reduced in DNP tissues compared with control tissues and SIRT1 expression levels decreased with the increasing disease severity.

Expression levels of autophagy-associated LC3 II/I and beclin-1 in DNP tissues are lower compared with control tissues. To study how IVDD is associated with autophagy, LC3 II/I and beclin-1 mRNA and protein expression levels were detected with RT-qPCR and western blotting, respectively in DNP and control tissues. LC3 and beclin-1 mRNA expression levels in DNP tissues were significantly lower compared with control tissues $(\mathrm{P}<0.05$ and $\mathrm{P}<0.01$, respectively; Fig. 2A and B). Western blotting analysis indicated that LC3 II/I and beclin-1 protein expression levels in DNP tissues were also significantly lower compared with control tissues $(\mathrm{P}<0.05$; Fig. $2 \mathrm{C}$ and $\mathrm{D})$. Taken together, the aforementioned results indicated that the expression levels of autophagy-associated LC3 II/I and beclin-1 in DNP tissues are lower compared with control tissues.

SIRT1 regulates LC3 II/I and beclin-1 expression in nucleus pulposus cells. To investigate the mechanism by which SIRT1 regulates IVDD, nucleus pulposus cells were treated with SIRT1 agonist resveratrol or SIRT1 inhibitor nicotinamide and SIRT1, LC3 II/I and beclin-1 expression levels were determined using RT-qPCR and western blotting. SIRT1 mRNA and protein were significantly elevated for the resveratrol group compared with the control group $(\mathrm{P}<0.05$ and $\mathrm{P}<0.01$, respectively; Fig. $3 \mathrm{~A}$ and $\mathrm{B})$. In addition, SIRT1 mRNA and protein expression levels were significantly lower in the nicotinamide group compared with the control group $(\mathrm{P}<0.05$; Fig. $3 \mathrm{~A}$ and $\mathrm{B})$. LC3 II/I mRNA and protein expression levels were significantly higher in the resveratrol group compared with the control group $(\mathrm{P}<0.01$ and $\mathrm{P}<0.05$, respectively; Fig. $3 \mathrm{C}$ and $\mathrm{D}$ ) and were significantly lower in the nicotinamide group when compared with the control group $(\mathrm{P}<0.05$; Fig. $3 \mathrm{C}$ and $\mathrm{D})$. Beclin-1 mRNA and protein expression levels were significantly elevated in the resveratrol group compared with the control group $(\mathrm{P}<0.01$ and $\mathrm{P}<0.05$, respectively; Fig. $3 \mathrm{E}$ and $\mathrm{F}$ ) and were significantly lower in the 

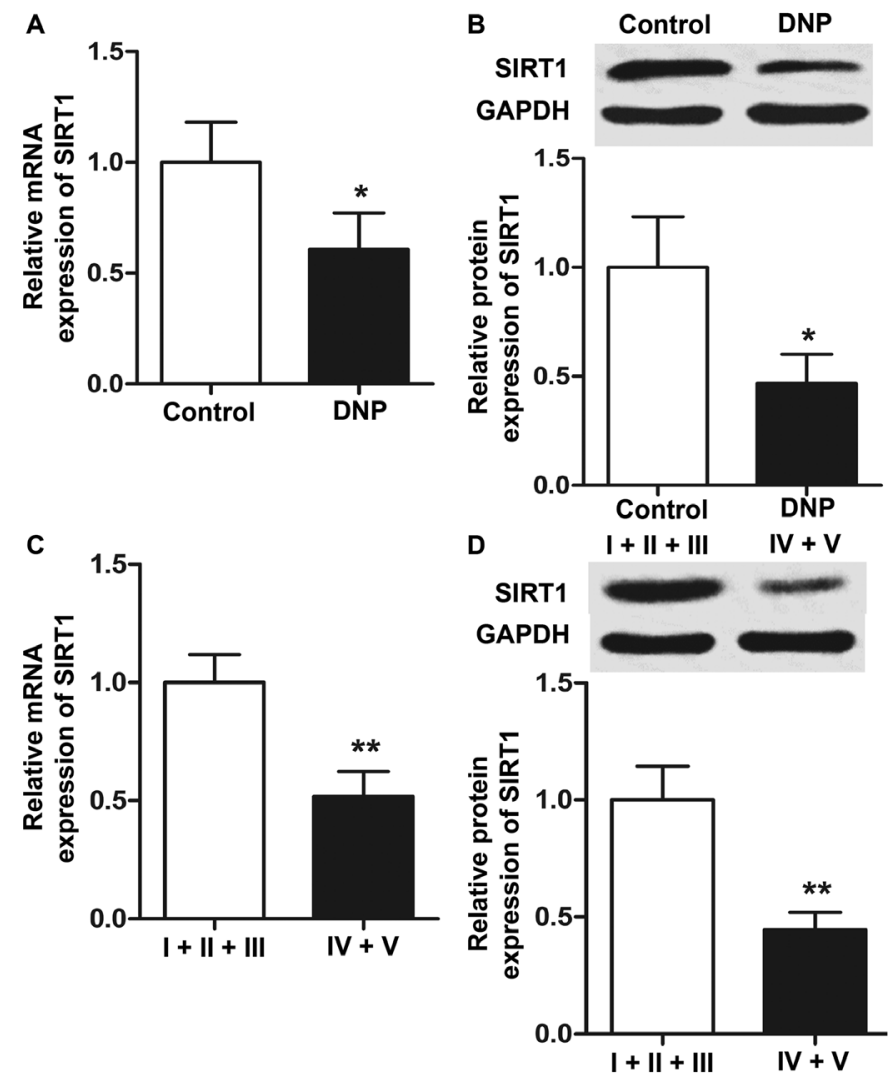

Figure 1. Expression of SIRT1 in nucleus pulposus tissues. (A) SIRT1 mRNA and (B) protein expression levels in control (normal nucleus pulposus) and DNP tissues. "P $<0.05$ compared to control. (C) mRNA expression levels and (D) protein expression levels of SIRT1 in DNP tissues from IVDD patients at grades I, II and III and IVDD patients at grades IV and V. ${ }^{* *} \mathrm{P}<0.01$ compared with IVDD patients at grades I, II and III. RT-qPCR was used to measure mRNA levels and western blotting was used to assess protein expression. SIRT1, sirtuin 1; RT-q, reverse transcription-quantitative; DNP, degenerative nucleus pulposus IVDD, intervertebral disc degeneration.
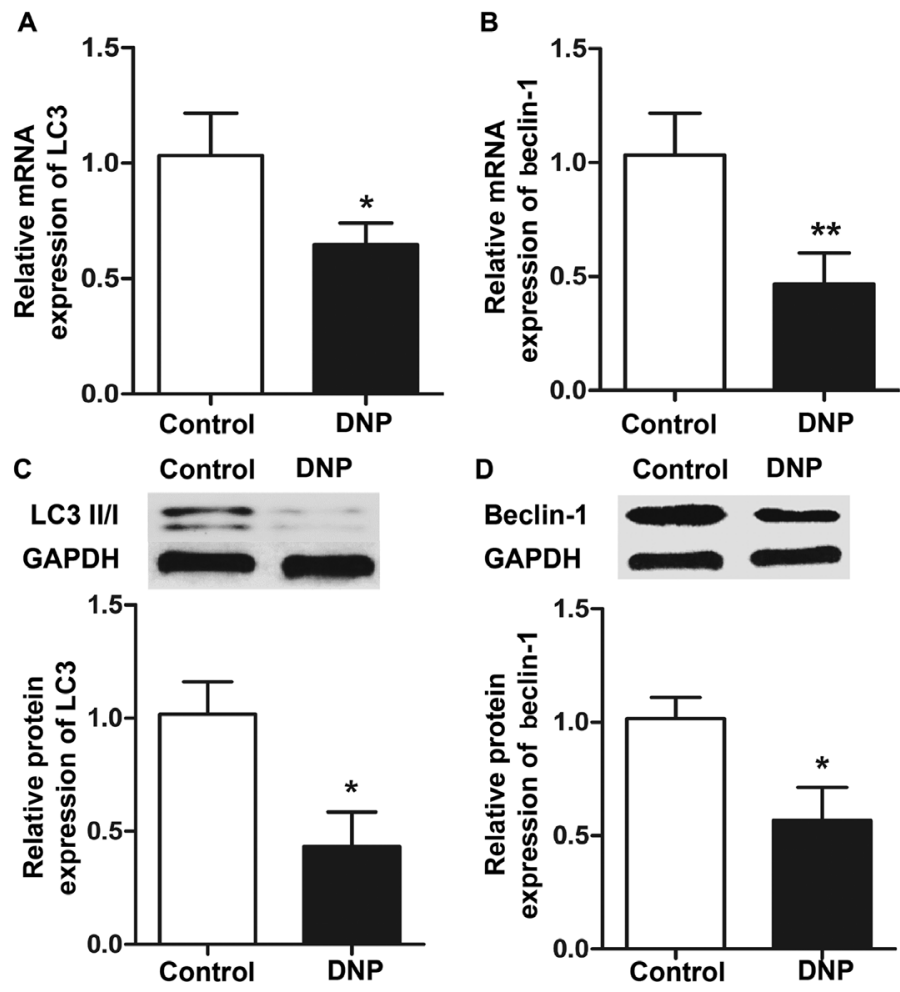

Figure 2. Expression of autophagy-associated genes in nucleus pulposus tissues. (A) LC3 and (B) beclin-1 mRNA expression levels in DNP tissues compared with control (normal nucleus pulposus) tissues. RT-qPCR was performed to determine the relative mRNA expression levels. ${ }^{*} \mathrm{P}<0.05$ and ${ }^{* * *} \mathrm{P}<0.01$ compared to control. (C) LC3 and (D) beclin-1 protein expression levels in the DNP tissues compared with control (normal nucleus pulposus) tissues. Western blotting was performed to measure the relative protein expression levels. " $\mathrm{P}<0.05$ compared to control. RT-q, reverse transcription-quantitative; DNP, degenerative nucleus pulposus. 
A

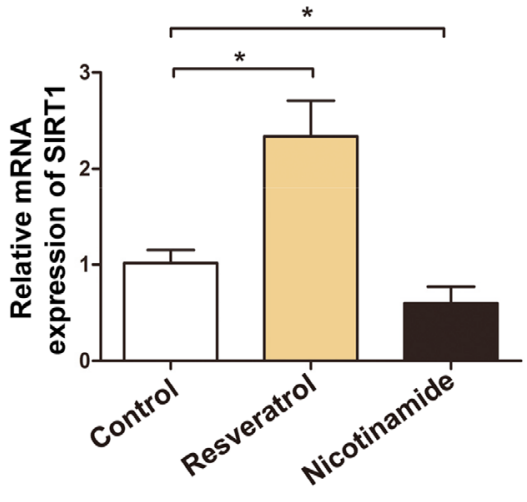

C

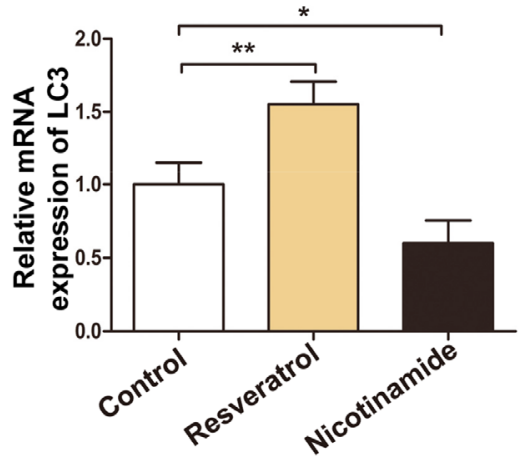

E

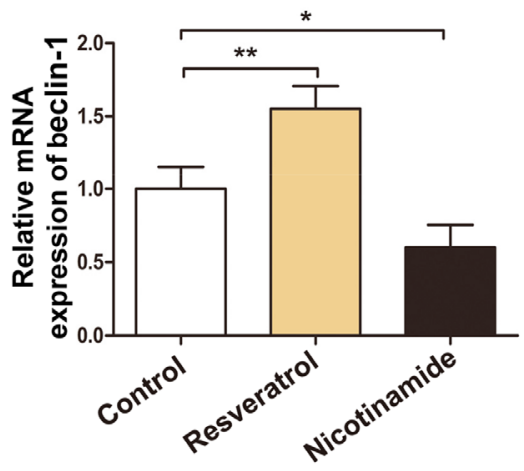

B

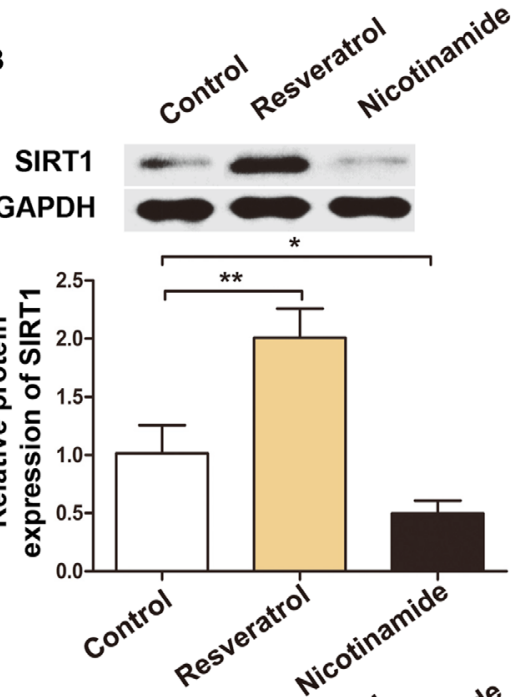

D

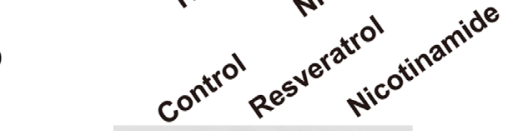

LC3 II/I

GAPDH

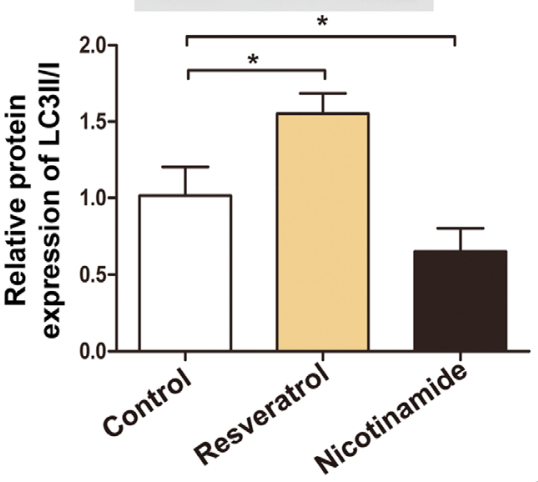

$\mathbf{F}$

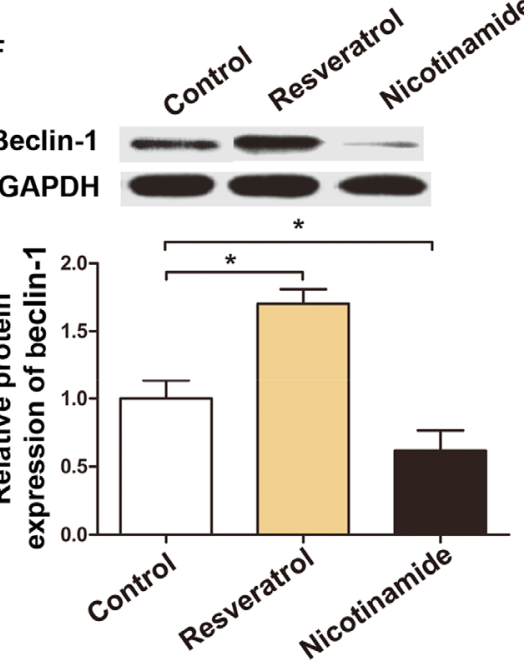

Figure 3. Effect of SIRT1 on the expression of autophagy-associated genes in nucleus pulposus cells. SIRT1 (A) mRNA and (B) protein expression levels in cells treated with resveratrol or nicotinamide. LC3 (C) mRNA and (D) protein expression levels in cells treated with resveratrol or nicotinamide. Beclin 1 (E) mRNA and (F) protein expression levels in cells treated with resveratrol or nicotinamide. ${ }^{*} \mathrm{P}<0.05$ and ${ }^{* *} \mathrm{P}<0.01$. RT-qPCR was used to measure mRNA levels and western blotting was used to assess protein expression. Resveratrol; SIRT1 agonist; nicotinamide; SIRT1 inhibitor; SIRT1, sirtuin 1; RT-q, reverse transcription-quantitative; control, cells treated with the solvent of the SIRT1 inhibitor.

nicotinamide group compared with the control group $(\mathrm{P}<0.05$; Fig. 3E and F). In summary, the aforementioned results indicated that SIRT1 regulates LC3 II/I and beclin-1 expression in nucleus pulposus cells.
Nampt/NAD $D^{+}$SIRT1 pathway participates in the process of IVDD by regulating autophagy of nucleus pulposus cells. To explore the mechanism of action of Nampt and SIRT1 in IVDD, Nampt inhibitor FK866 was used to treat nucleus 
A
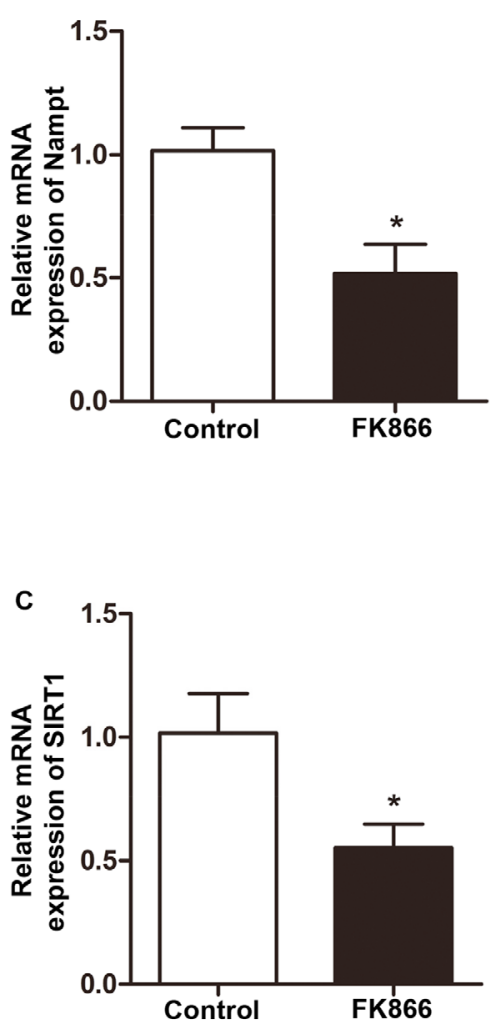

E

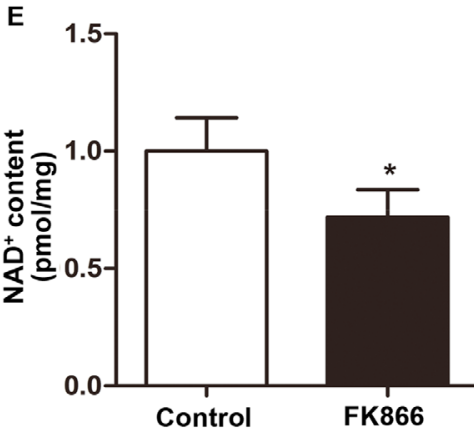

B
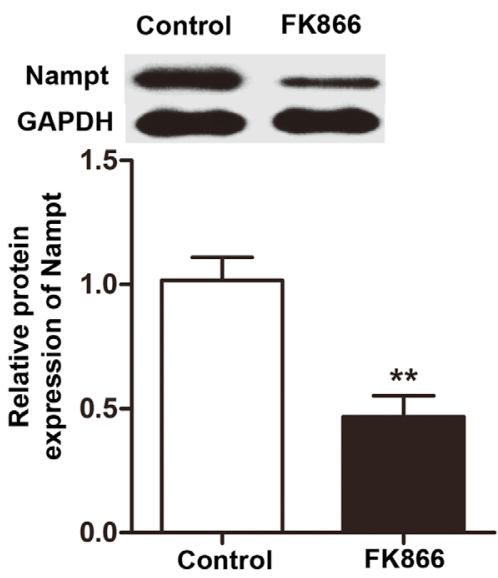

D Control FK866
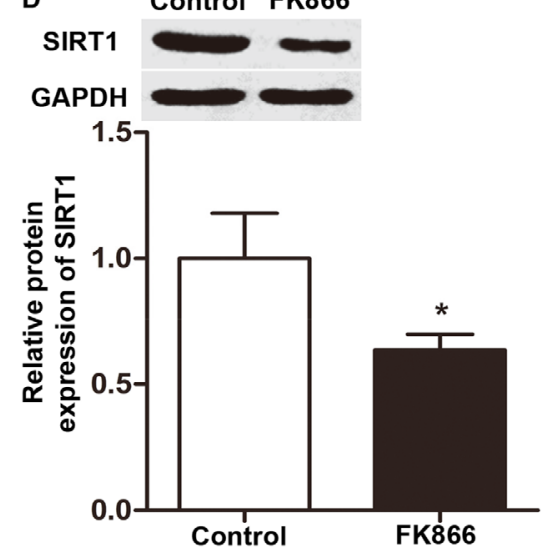

F Control FK866 Control FK866

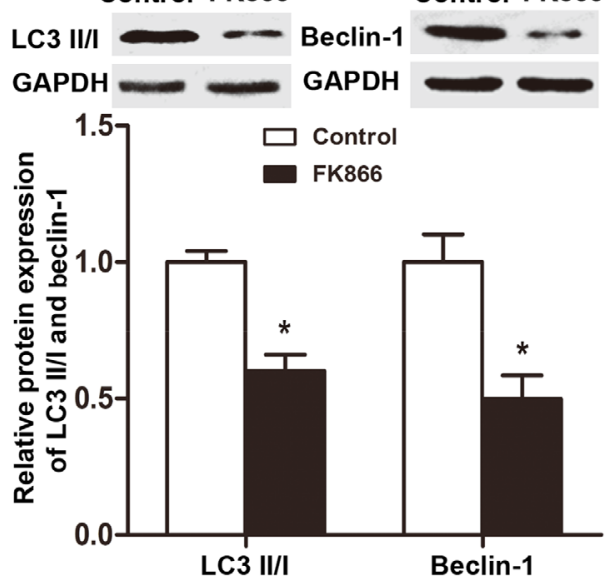

Figure 4. Nampt/NAD $/$ SIRT1 pathway in nucleus pulposus cells. Nampt (A) mRNA and (B) protein expression levels in control cells and cells treated with FK866. (C) mRNA expression levels and (D) protein expression levels of SIRT1 in control cells and cells treated with FK866. (E) NAD ${ }^{+}$contents in control cells and cells treated with FK866 (F) LC3II/I and beclin-1 protein expression levels in in control cells and cells treated with FK866. "P<0.05 and ${ }^{* *} \mathrm{P}<0.01$ compared with the control group. RT-qPCR was used to measure mRNA levels and western blotting was used to assess protein expression and NAD" contents were detected with the $\mathrm{NAD}^{+}$concentration determination kit. FK866, Nampt inhibitor; control group, untreated cells; Nampt, nicotinamide phosphoribosyl transferase; NAD, nicotinamide adenine dinucleotide; SIRT1, sirtuin 1; RT-q, reverse transcription-quantitative.

pulposus cells. Nampt mRNA and protein expression levels were significantly lower in FK866 group compared with the control group $(\mathrm{P}<0.05$ and $\mathrm{P}<0.01$, respectively; Fig. 4A and $\mathrm{B})$. The mRNA and protein expression levels of SIRT1 were significantly lower in the FK866 group compared with the control group $(\mathrm{P}<0.05$; Fig. $4 \mathrm{C}$ and $\mathrm{D})$. In addition, $\mathrm{NAD}^{+}$ levels were significantly lower in the FK866 group compared with the control group ( $\mathrm{P}<0.05$; Fig. 4E). Notably, protein expression levels of beclin-1 and LC3II/I were significantly lower in the FK866 group compared with the control group $(\mathrm{P}<0.05$; Fig. 4F). The aforementioned findings suggested that the Nampt/NAD ${ }^{+} / \mathrm{SIRT} 1$ pathway participates in the process of IVDD by regulating autophagy of nucleus pulposus cells.

\section{Discussion}

The present study demonstrated that SIRT1 mRNA and protein expression levels in DNP were significantly declined 
compared with those in normal nucleus pulposus tissues suggesting an association between SIRT1 and IVDD. The present study, also found that the expression of LC3 II/I and beclin-1 in DNP tissues was significantly lower compared with that in control tissues. After treating nucleus pulposus cells with SIRT1 agonist resveratrol, the mRNA and protein expression levels of SIRT1, LC3II/I and beclin-1 were increased and autophagy-related genes were altered. After treating nucleus pulposus cells with SIRT1 inhibitor nicotinamide, the expression levels of SIRT1, LC3II/I and beclin-1 reduced and autophagy of degenerative nucleus pulposus cells was probably reduced. The aforementioned findings of the present study have shed light on the effect of SIRT1 in autophagy.

Autophagy is a process in which cells phagocytose and degrade their own cytoplasm and organelles via the lysosomal system, which exerts important functions in the development and growth of organisms, cell homeostasis, maturation and differentiation (24). According to the different pathways of cell material transport to lysosomes, autophagy is classified into large autophagy, small autophagy and chaperone-mediated autophagy (CMA) (24). It is reported that SIRT1 may be involved in CMA by regulating autophagy of nucleus pulposus cells via the Akt/mTOR or Erk/mTOR pathways (25). Autophagy is also involved in the pathological processes of numerous diseases, such as cardiovascular diseases, nervous system diseases, metabolic diseases and cancers $(25,26)$ Hence, autophagy exerts important effects in the maintenance of cellular survival and homeostasis (26). It has been reported that the enhanced autophagy inhibits apoptosis and protects nerve cells (27). In addition, autophagy activity of articular cartilage in severe osteoarthritis was significantly reduced and apoptotic rate was enhanced compared with that in normal subjects; in addition, autophagy reduced the degeneration of annulus fibrosus by inhibiting apoptosis (28). An animal study has demonstrated that autophagy activity of nucleus pulposus cells was gradually increased with the degeneration of the caudal intervertebral disc in rats (29). The findings of the aforementioned studies suggest that autophagy may be important in the pathogenesis and development of IVDD. MMP-13 is another major catabolic enzyme that leads to intervertebral disc degeneration (29). Autophagy is a protective mechanism in normal articular cartilage, and autophagy activity and related proteins such as LC3 II/I and beclin-1 are significantly reduced in degenerative articular cartilage (30). In the present study, LC3 II/I and beclin-1 expression levels were assessed to reflect autophagy. The findings of the present study demonstrated that the expression of autophagy-associated proteins LC3 II/I and beclin-1 in DNP tissues of patients with IVDD was significantly lower compared with control tissues, suggesting that autophagy may be used to degrade damaged and dysfunctional organelles and macromolecules to protect nucleus pulposus tissues.

$\mathrm{NAD}^{+}$is a coenzyme of SIRT1 and the activation and biological activity of SIRT1 are both dependent on $\mathrm{NAD}^{+}(30)$. The synthesis of $\mathrm{NAD}^{+}$occurs via 3 pathways, including salvage pathway, de novo pathway and Preiss-Handler pathway, among which the salvage pathway is the major one $(18,19)$. Nampt is a rate-limiting enzyme for $\mathrm{NAD}^{+}$ remedial synthetic pathway (31). Under physiological conditions, Nampt can be expressed in a number of cells in the body, such as cardiomyocytes, nerve cells and fat cells (30). Nampt has been implicated in regulating the processes of autophagy and apoptosis. For example, Nampt as an inhibitor of apoptosis in neutrophils can delay the induction of neutrophil apoptosis by inflammatory factors such as IGF-1, TNF- $\alpha$ and NF- $\kappa \mathrm{B}$ (32). A previous study demonstrated that Nampt may protect cardiomyocytes by inducing autophagy, reducing oxidative stress and eliminating damaged mitochondria (33). A study on cerebral ischemia demonstrated that Nampt can improve the survival rate of neurons by inducing autophagy of neurons (34). In addition, treatment of chondrocytes with Nampt can induce high expression of MMP-13, A Disintegrin and Metalloproteinase with Thrombospondin motifs (ADAMTS)-4 and ADAMTS-5 (35). The degradation of extracellular matrix and the loss of proteoglycan are important reasons for joint degeneration and ADAMTS- 4 and ADAMTS-5 are important degradation enzymes of extracellular matrix proteoglycans in joints (35). Therefore, elevated expression of these proteins implies potential joint degeneration. The findings of the aforementioned studies suggested that Nampt may participate in numerous physiological and disease processes through autophagy.

Treatments IVDD mainly include traditional surgical replacement and biological treatment such as cell therapy (36). Although at present, the replacement of intervertebral disc prosthesis can restore the flexion and extension ability of patients, an improved clinical effect compared with spinal fusion was not observed (37). Although SIRT1 agonists and inhibitors are not SIRT1-specific, a number of studies have proven their effects on SIRT1 $(38,39)$, and they can be used as effective means to regulate SIRT1 expression (40). The findings of the present study indirectly demonstrated the effectiveness of SIRT1 agonists and inhibitors in regulating SIRT1 expression by regulating its upstream molecule Nampt. The development prospect of biological treatment of IVDD is very promising (7). The findings of the present study suggested that SIRT1 may serve a protective role in IVDD by regulating autophagy. The present study still has its limitation because it did not explain how SIRT1 regulated downstream molecules to participate in IVDD and the interaction between the molecules, which still need further studies. Future studies should develop specific drugs that target SIRT1 and this may lead to a new direction for the treatment of IVDD. Although the findings of the present study suggested that SIRT1-regulated downstream autophagy signaling pathway served a key role in IVDD, the detailed pathogenesis of IVDD and the roles of other key molecules in autophagy signaling pathway in IVDD need to be explored further by future studies.

In conclusion, the findings of the present study demonstrated that SIRT1 may be involved in the development of IVDD via the Nampt/NAD ${ }^{+} / \mathrm{SIRT} 1$ pathway that regulates autophagy of nucleus pulposus cells.

\section{Acknowledgements}

Not applicable.

\section{Funding}

No funding was received. 


\section{Availability of data and materials}

The datasets used and/or analyzed during the current study are available from the corresponding author on reasonable request.

\section{Authors' contributions}

QS and XZ contributed to the design of the study. QS and LX performed the experiments and analyzed the data. QS and XZ interpreted results and drafted the manuscript. QS and LX confirmed the authenticity of all the raw data. All authors have read and approved the manuscript.

\section{Ethics approval and consent to participate}

The present study was approved by the Ethics Committee of Jinzhou Medical University (Jinzhou, China). Written informed consent was obtained from all patients or their families.

\section{Patient consent for publication}

Not applicable.

\section{Competing interests}

The authors declare that they have no competing interests.

\section{References}

1. Becker A, Held H, Redaelli M, Chenot JF, Leonhardt C, Keller S, Baum E, Pfingsten M, Hildebrandt J, Basler HD, et al: Implementation of a guideline for low back pain management in primary care: A cost-effectiveness analysis. Spine 37: 701-710, 2012.

2. Mirza SK and Deyo RA: Systematic review of randomized trials comparing lumbar fusion surgery to nonoperative care for treatment of chronic back pain. Spine 32: 816-823, 2007.

3. Hanley EN Jr, Herkowitz HN, Kirkpatrick JS, Wang JC, Chen MN and Kang JD: Debating the value of spine surgery. J Bone Joint Surg Am 92: 1293-1304, 2010.

4. Fassett DR, Kurd MF and Vaccaro AR: Biologic solutions for degenerative disk disease. J Spinal Disord Tech 22: 297-308, 2009.

5. Miller JA, Schmatz C and Schultz AB: Lumbar disc degeneration: Correlation with age, sex, and spine level in 600 autopsy specimens. Spine 13: 173-178, 1988

6. König SA, Goldammer A and Vitzthum HE: Anatomical data on the craniocervical junction and their correlation with degenerative changes in 30 cadaveric specimens. J Neurosurg Spine 3 : 379-385, 2005.

7. Luoma K, Riihimäki H, Luukkonen $\mathrm{R}$, Raininko $\mathrm{R}$, Viikari-Juntura $\mathrm{E}$ and Lamminen A: Low back pain in relation to lumbar disc degeneration. Spine 25: 487-492, 2000.

8. Leibiger IB and Berggren PO: Sirt1: A metabolic master switch that modulates lifespan. Nat Med 12: 34-36, 2006.

9. Livak KJ and Schmittgen TD: Analysis of relative gene expression data using real-time quantitative PCR and the 2(-Delta Delta C(T)) Method. Methods 25: 402-408, 2001.

10. Akasaki Y, Alvarez-Garcia O, Saito M, Caramés B, Iwamoto Y and Lotz MK: FoxO transcription factors support oxidative stress resistance in human chondrocytes. Arthritis Rheumatol 66: 3349-3358, 2014.

11. Takayama K, Ishida K, Matsushita T, Fujita N, Hayashi S, Sasaki K, Tei K, Kubo S, Matsumoto T, Fujioka H, et al: SIRT1 regulation of apoptosis of human chondrocytes. Arthritis Rheum 60: 2731-2740, 2009.

12. Ulakcsai Z, Bagaméry F, Vincze I, Szökő É and Tábi T: Protective effect of resveratrol against caspase 3 activation in primary mouse fibroblasts. Croat Med J 56: 78-84, 2015.
13. Almeida M and Porter RM: Sirtuins and FoxOs in osteoporosis and osteoarthritis. Bone 121: 284-292, 2019.

14. Ma CH, Chiua YC, Wu CH, Jou IM, Tu YK, Hung CH, Hsieh PL and Tsai KL: Homocysteine causes dysfunction of chondrocytes and oxidative stress through repression of SIRT1/AMPK pathway: A possible link between hyperhomocysteinemia and osteoarthritis. Redox Biol 15: 504-512, 2018.

15. Yang LJ, Chen Y, He J, Yi S, Wen L, Zhao S and Cui GH: Effects of gambogic acid on the activation of caspase-3 and downregulation of SIRT1 in RPMI-8226 multiple myeloma cells via the accumulation of ROS. Oncol Lett 3: 1159-1165, 2012.

16. Chauhan D, Bandi M, Singh AV, Ray A, Raje N, Richardson P and Anderson KC: Preclinical evaluation of a novel SIRT1 modulator SRT1720 in multiple myeloma cells. Br J Haematol 155: 588-598, 2011.

17. Bi TQ, Che XM, Liao XH, Zhang DJ, Long HL, Li HJ and Zhao W: Overexpression of Nampt in gastric cancer and chemopotentiating effects of the Nampt inhibitor FK866 in combination with fluorouracil. Oncol Rep 26: 1251-1257, 2011.

18. Benedict C, Shostak A,Lange T,Brooks SJ,Schiöth HB,Schultes B, Born J, Oster H and Hallschmid M: Diurnal rhythm of circulating nicotinamide phosphoribosyltransferase (Nampt/visfatin/PBEF): Impact of sleep loss and relation to glucose metabolism. J Clin Endocrinol Metab 97: E218-E222, 2012

19. Friebe D, Löffler D, Schönberg M, Bernhard F, Büttner P, Landgraf K, Kiess W and Körner A: Impact of metabolic regulators on the expression of the obesity associated genes FTO and NAMPT in human preadipocytes and adipocytes. PLoS One 6: e19526, 2011.

20. Laiguillon MC, Houard X, Bougault C, Gosset M, Nourissat G, Sautet A, Jacques C, Berenbaum F and Sellam J: Expression and function of visfatin (Nampt), an adipokine-enzyme involved in inflammatory pathways of osteoarthritis. Arthritis Res Ther 16: R38, 2014.

21. Guo JY, Li F, Wen YB, Cui HX, Guo ML, Zhang L, Zhang YF, Guo YJ and Guo YX: Melatonin inhibits Sirt1-dependent NAMPT and NFAT5 signaling in chondrocytes to attenuate osteoarthritis. Oncotarget 8: 55967-55983, 2017.

22. Nowell M, Evans L and Williams A: PBEF/NAMPT/visfatin: A promising drug target for treating rheumatoid arthritis? Future Med Chem 4: 751-769, 2012.

23. Franco-Trepat E,Alonso-Pérez A, Guillán-FrescoM,Jorge-Mora A, Gualillo O, Gómez-Reino JJ and Gómez Bahamonde R: Visfatin as a therapeutic target for rheumatoid arthritis. Expert Opin Ther Targets 23: 607-618, 2019.

24. Levine B and Kroemer G: Autophagy in the pathogenesis of disease. Cell 132: 27-42, 2008.

25. Glick D, Barth S and Macleod KF: Autophagy: Cellular and molecular mechanisms. J Pathol 221: 3-12, 2010.

26. Rabinowitz JD and White E: Autophagy and metabolism. Science 330: 1344-1348, 2010.

27. Wu JJ, Quijano C, Wang J and Finkel T: Metabolism meets autophagy. Cell Cycle 9: 4780-4781, 2010.

28. MetcalfDJ,García-Arencibia M,Hochfeld WEand Rubinsztein DC: Autophagy and misfolded proteins in neurodegeneration. Exp Neurol 238: 22-28, 2012.

29. Caramés B, Taniguchi N, Otsuki S, Blanco FJ and Lotz M: Autophagy is a protective mechanism in normal cartilage, and its aging-related loss is linked with cell death and osteoarthritis. Arthritis Rheum 62: 791-801, 2010.

30. Almonte-Becerril M, Navarro-Garcia F, Gonzalez-Robles A, Vega-Lopez MA, Lavalle C and Kouri JB: Cell death of chondrocytes is a combination between apoptosis and autophagy during the pathogenesis of Osteoarthritis within an experimental model. Apoptosis 15: 631-638, 2010.

31. Imai S and Yoshino J: The importance of NAMPT/NAD/SIRT1 in the systemic regulation of metabolism and ageing. Diabetes Obes Metab 15 (Suppl 3): 26-33, 2013.

32. Imai SI: The NAD World 2.0: The importance of the inter-tissue communication mediated by NAMPT/NAD ${ }^{+} /$SIRT1 in mammalian aging and longevity control. NPJ Syst Biol Appl 2: 16018, 2016.

33. Yang P, Zhang L, Shi QJ, Lu YB, Wu M, Wei EQ and Zhang WP: Nicotinamide phosphoribosyltransferase inhibitor APO866 induces C6 glioblastoma cell death via autophagy. Pharmazie 70: 650-655, 2015.

34. Hsu CP, Hariharan N, Alcendor RR, Oka S and Sadoshima J: Nicotinamide phosphoribosyltransferase regulates cell survival through autophagy in cardiomyocytes. Autophagy 5: 1229-1231, 2009. 
35. Wang P, Guan YF, Du H, Zhai QW, Su DF and Miao CY: Induction of autophagy contributes to the neuroprotection of nicotinamide phosphoribosyltransferase in cerebral ischemia. Autophagy 8: 77-87, 2012.

36. Won Y, Shin Y, Chun CH, Cho Y, Ha CW, Kim JH and Chun JS Pleiotropic roles of metallothioneins as regulators of chondrocyte apoptosis and catabolic and anabolic pathways during osteoarthritis pathogenesis. Ann Rheum Dis 75: 2045-2052, 2016.

37. Heider FC, Mayer HM and Siepe CJ: Lumbar disc replacement: Update. J Neurosurg Sci 59: 169-180, 2015.

38. Veruva SY, Steinbeck MJ, Toth J, Alexander DD and Kurtz SM: Which design and biomaterial factors affect clinical wear performance of total disc replacements? A systematic review. Clin Orthop Relat Res 472: 3759-3769, 2014.
39. Zhao Y, Luo P, Guo Q, Li S, Zhang L, Zhao M, Xu H, Yang Y, Poon W and Fei Z: Interactions between SIRT1 and MAPK/ERK regulate neuronal apoptosis induced by traumatic brain injury in vitro and in vivo. Exp Neurol 237: 489-498, 2012.

40. Hong EH, Yun HS, Kim J, Um HD, Lee KH, Kang CM, Lee SJ, Chun JS and Hwang SG: Nicotinamide phosphoribosyltransferase is essential for interleukin-1beta-mediated dedifferentiation of articular chondrocytes via SIRT1 and extracellular signal-regulated kinase (ERK) complex signaling. J Biol Chem 286: 28619-28631, 2011.

This work is licensed under a Creative Commons Attribution-NonCommercial-NoDerivatives 4.0 International (CC BY-NC-ND 4.0) License. 